\title{
Guillain-Barré syndrome associated with COVID-19: an atypical, late-onset presentation
}

\author{
Elizabeth W. Fletman ${ }^{1}$ (D) Natalie Stumpf ${ }^{2} \cdot$ Jan Kalimullah $^{3} \cdot$ Noah Levinson $^{3} \cdot$ Anahita Deboo $^{3}$
}

Received: 10 December 2020 / Accepted: 17 May 2021 / Published online: 22 May 2021

(C) Fondazione Società Italiana di Neurologia 2021

\section{Dear Editor,}

Guillain-Barré syndrome (GBS) represents a diverse group of acute immune-mediated polyradiculoneuropathies associated with a variety of infectious illnesses, including, severe acute respiratory distress syndrome - coronavirus-2 (SARSCoV-2). Multiple subtypes of GBS have been associated with coronavirus disease-2019 (COVID-19) infections. Previously reported COVID-19-associated GBS demonstrates a range of 2-33 days from COVID-19 infection to neurological symptom onset [1]. We report a case of GBS presenting approximately 100 days following initial hospitalization with COVID-19.

A 67-year-old female presented to an outpatient neurology clinic with bilateral lower extremity ascending numbness and weakness 100 days following initial presentation with COVID-19-associated pneumonia. Initial COVID-19 symptoms included fever, cough, shortness of breath, nausea, and one episode of emesis. During initial hospitalization for COVID-19, the patient was admitted to the intensive care unit,

Elizabeth W. Fletman

elizabethfl@pcom.edu

Natalie Stumpf

tuj14014@temple.edu

Jan Kalimullah

Kalimullah.Jan@tuhs.temple.edu

Noah Levinson

noah.levinson@tuhs.temple.edu

Anahita Deboo

Anahita.Deboo@tuhs.temple.edu

1 Philadelphia College of Osteopathic Medicine, 4170 City Ave, Philadelphia, PA 19131, USA

2 Lewis Katz School of Medicine at Temple University Hospital, 3509 N Broad St 9th floor, Philadelphia, PA 19140, USA

3 Department of Neurology, Temple University Hospital, $3401 \mathrm{~N}$ Broad St, Philadelphia, PA 19140, USA placed on high-flow nasal cannula, and treated with steroids, remdesivir, tocilizumab, convalescent plasma, and anakinra. Nasopharyngeal swabs tested positive for 34 days. After a 47day hospital stay, the patient was discharged to a long-term acute care facility on a 10 -day course of prednisone $30 \mathrm{mg}$. At the time of discharge, she required assistance to stand and had decreased balance, strength, and range of motion.

The patient presented 71 days from last positive COVID19 test, reporting 3 weeks of bilateral plantar paresthesia which progressed in an asymmetric, ascending pattern. She developed bilateral foot drop and numbness in the fifth digits of both hands.

On exam, the patient had bilateral proximal and distal leg weakness, left greater than right. Sensation to cold was diminished in a length-dependent fashion distal to the knees, with greater deficit on the left than on the right leg. In the arms, sensation to cold was also diminished in a length-dependent fashion distal to the upper forearms, and there was decreased sensation over the fifth and medial one half of the fourth digit on the left. Vibration sense was absent at the toes and severely diminished at the ankles, but intact at the fingers. Ankle deep tendon reflexes were absent with flexor plantar responses bilaterally. She had no cranial nerve abnormalities (Table 1).

The patient was admitted to the inpatient neurology service. CSF analysis showed albuminocytological dissociation

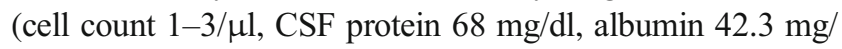
dl). CSF cytology showed few single plasmacytoid cells with a background of red blood cells, monocytes, and neutrophils. Serum ganglioside antibody assays were negative for antiGM1, anti-GM1b, anti-GD1a, anti-GD1b.

EMG/NCS demonstrated a severe, sensory motor polyneuropathy, or polyradiculoneuropathy affecting the legs greater than the arms that was primarily axonal with active denervation. Specifically, sensory and motor response amplitudes were absent or markedly diminished in the legs symmetrically. The arm showed normal or mildly diminished sensory and motor amplitudes. Conduction velocities and f-wave latencies were normal or mildly slowed. There was no 
Table 1 Neurological examination findings in patient on admission and prior to hospital discharge

\begin{tabular}{|c|c|c|}
\hline & Exam findings on admission & Exam findings prior to discharge \\
\hline \multirow[t]{4}{*}{ Motor } & Right UE: proximal 4+, distal 4 & Bilateral UE: proximal 5, distal 4+ \\
\hline & Left UE: proximal 4, distal 4- & \\
\hline & Right LE: proximal 4, distal 3 dorsiflexion, 4 plantarflexion & $\begin{array}{l}\text { Right LE: proximal 4+, distal } 3 \text { dorsiflexion } \\
\text { 4+ plantarflexion }\end{array}$ \\
\hline & Left LE: proximal 4-, distal 1 dorsiflexion, 2-3 plantarflexion & $\begin{array}{l}\text { Left LE: proximal 4+, distal } 1 \text { dorsiflexion, } \\
\text { 4- plantarflexion }\end{array}$ \\
\hline Sensory & $\begin{array}{l}\text { Cold sensation: diminished distal to knees and upper forearms. } \\
\text { Left hand decreased over the fifth and medial one half of the fourth digit. } \\
\text { Vibration: absent at the toes and severely diminished at the ankles, } \\
\text { but intact at the fingers. }\end{array}$ & $\begin{array}{l}\text { Light touch: diminished distal to lower shin. } \\
\text { Left hand decreased over fifth digit. }\end{array}$ \\
\hline \multirow[t]{4}{*}{ Deep tendon reflexes } & Biceps and triceps 2 and symmetric & Biceps and triceps $2+$ and symmetric \\
\hline & Right patellar 1, left patellar absent & Bilateral patellar 1+ with Jendrassik \\
\hline & Bilateral achilles absent & Bilateral achilles absent \\
\hline & Bilateral plantar responses flexor & Bilateral plantar responses flexor \\
\hline
\end{tabular}

Abbreviations: $U E$, upper extremity; $L E$, lower extremity

conduction block. Fibrillations and positive sharp waves were seen in proximal and distal leg muscles with reduced recruitment and interference patterns but no motor unit morphology changes, supporting acute denervation.

The patient received a 5-day course of IVIG. After an 11day hospitalization, she was discharged with improvement in the motor and sensory examination as outlined in Table 1.

\section{Discussion}

GBS is a heterogenous group of acute immune-mediated generalized polyradiculoneuropathies commonly precipitated by an infectious illness. GBS has been associated with Campylobacter jejuni infection, Cytomegalovirus, EpsteinBarr virus, HIV, influenza, Zika virus, and most recently SARS-CoV-2 [1,2]. One proposed mechanism of GBS is that antecedent infection triggers antibodies, which, in turn, crossreact with peripheral nerves through immune system molecular mimicry $[1,3]$. Major subtypes of GBS include acute inflammatory demyelinating polyneuropathy (AIDP), acute motor axonal neuropathy (AMAN), acute motor sensory axonal neuropathy (AMSAN), Miller Fisher syndrome (MFS), and its variants [4].

Clinical presentation varies in terms of the distribution and extent of weakness, sensory symptoms, ataxia and cranial nerve deficits [5]. Common presenting symptoms of nonCOVID-19-associated GBS include paresthesia, numbness, and ascending weakness $[3,4]$. Previous literature reports intervals of COVID-19 symptom onset to neurological symptom onset ranging between 2 and 33 days. Common neurological symptoms include sensory changes alone $(72.2 \%)$ or in conjunction with paraparesis or tetraparesis $(65.2 \%)$ [1]. A previous systematic review reported that out of 72 GBS cases,
70 patients presented with symptomatic COVID-19 of variable severity [1].

Serologic features differ between subtypes; IgG autoantibodies against GM1 or GD1a are strongly associated with AMAN and AMSAN. Anti-GQ1b antibodies are strongly associated with MFS and its variants [3]. In comparison, previous literature regarding COVID-19-associated GBS reported negative serum ganglioside antibodies (one in equivocal range) in 33 out of 35 cases. One patient with MFS and one patient with classical sensorimotor GBS tested positive for anti-GD1b and anti-GM1 respectively [1].

The trademark CSF finding is albuminocytological dissociation, which is seen in $64 \%$ of non-COVID-19-associated GBS cases [5], and $71.2 \%$ of COVID-19-associated GBS cases [1]. Electrophysiological data differentiates demyelinating from axonal subtypes [5]. EMG data of 62 patients with COVID-19-associated GBS showed $77.4 \%$ of cases presented with a demyelinating polyradiculoneuropathy, $14.5 \%$ demonstrated axonal damage, and $8.1 \%$ showed mixed pattern [1]. EMG data further showed that $81.8 \%$ of patients met criteria for AIDP, $12.7 \%$ AMSAN, and 5.4\% AMAN [1].

Our patient had an atypical presentation of COVID-19associated GBS due to the 100-day interval between COVID-19 hospitalization and neurological symptom onset. Furthermore, our patient presented with an AMSAN, whereas the majority of reported cases present with a demyelinating polyradiculoneuropathy $[1,6,7]$. A previous case report details late onset GBS 53 days following SARS-CoV-2 infection [8]. Our patient demonstrated a distinctly longer latency period between COVID-19 symptoms and GBS than previously reported cases. While it is probable that the patient's GBS was triggered by the SARS-CoV-2 infection, it remains possible that the two events were unrelated. All serum ganglioside antibodies were absent in our patient, which is consistent with 
previously reported cases of COVID-19-associated GBS with axonal variant $[1,6]$. To our knowledge, previous cases of GBS following infection, including after COVID-19, have not been described greater than 3 months following infection. We report a patient with GBS axonal variant 100 days following COVID-19 in an effort to add to the understanding of COVID-19-associated neurological complications. So that we can further develop this understanding, we hope that others will continue to report potential long-term sequela of COVID19.

Abbreviations GBS, Guillain-Barré syndrome; SARS-CoV-2, severe acute respiratory distress syndrome - coronavirus-2; COVID-19, coronavirus disease-2019; CSF, cerebrospinal fluid; EMG, electromyography; NCS, nerve conduction studies; IVIG, intravenous immunoglobulin; AIDP, acute inflammatory demyelinating polyneuropathy; AMAN, acute motor axonal neuropathy; AMSAN, acute motor sensory axonal neuropathy; MFS, Miller Fisher syndrome

Availability of data and material All available data was presented in the manuscript.

Code availability Not applicable

Author contribution All authors contributed to the organization, data collection and analysis, drafting and review of the manuscript.

\section{Declarations}

Ethical approval All institutional ethical guidelines were followed throughout the study.
Conflict of interest Informed consent was obtained from patient.

\section{References}

1. Abu-Rumeileh S, Abdelhak A, Foschi M, Tumani H, Otto M (2020) Guillain-Barré syndrome spectrum associated with COVID-19: an up-to-date systematic review of 73 cases. J Neurol Epub 268(4): 1133-1170. https://doi.org/10.1007/s00415-020-10124-x

2. Caress JB, Castoro RJ, Simmons Z et al (2020) COVID-19-associated Guillain-Barré syndrome: the early pandemic experience. Muscle Nerve 62:485-491. https://doi.org/10.1002/mus.27024

3. Yuki N, Hartung HP (2012) Guillain-Barré syndrome. N Engl J Med 366:2294-2304. https://doi.org/10.1056/NEJMra1 114525

4. Cosi V, Versino M (2006) Guillain-Barré syndrome. Neurol Sci 27(suppl):S47-SS5. https://doi.org/10.1007/s10072-006-0548-4

5. Van den Berg B, Walgaard C, Drenthen J et al (2014) Guillain-Barré syndrome: pathogenesis, diagnosis, treatment and prognosis. Nat Rev Neurol 10:469-482. https://doi.org/10.1038/nrneurol.2014.121

6. Kaveh R (2020) Guillain-Barre syndrome during COVID-19 pandemic: an overview of the reports. Neurol Sci 41:3149-3156. https:// doi.org/10.1007/s10072-020-04693-y

7. Agosti E, Giorgianni A, Francesco D et al (2021) Is Guillain-Barré syndrome triggered by SARS-CoV-2? Case report and literature review. Neurol Sci 42:607-612. https://doi.org/10.1007/s10072-02004553-9

8. Raahimi MM, Kane A, Moore CEG, Alareed AW (2021) Late onset of Guillain-Barré syndrome following SARS-CoV-2 infection: part of 'long COVID-19 syndrome'. BMJ Case Rep 14:e240178. https:// doi.org/10.1136/bcr-2020-240178

Publisher's note Springer Nature remains neutral with regard to jurisdictional claims in published maps and institutional affiliations. 